The original publication is available at Sanz, C., Belaj, A., Tortosa, J.L. and Pérez, A.G. (2020). Comparative study of the content of phenolic compounds in olive fruits and leaves for possible use in breeding programs for the functional selection of olive cultivars. Acta Hortic. 1282, 11-18

DOI: 10.17660/ActaHortic.2020.1282.3

https://doi.org/10.17660/ActaHortic.2020.1282.3

\title{
Comparative study of the content of phenolic compounds in olive fruits and leaves for possible use in breeding programs for the functional selection of olive cultivars
}

\author{
C. Sanz ${ }^{1, a}$, A. Belaj², J.L. Tortosa1, and A.G. Pérez ${ }^{1}$ \\ 1 Instituto de la Grasa (CSIC), Sevilla, Spain; ${ }^{2}$ IFAPA, Centro Alameda del Obispo, Córdoba, Spain.
}

\begin{abstract}
Olive breeding has traditionally focused on the agronomic traits but the increasing number of scientific proofs supporting the positive impact of virgin olive oil (VOO) consumption on human health has made the functional quality of this product to be considered as an olive breeding target, particularly regarding the phenolic fraction of VOO. The presence of phenolic compounds in VOO is based mainly on the content of phenolic glucosides initially present in the olive fruit tissues and on the activity of hydrolytic and oxidative enzymes acting on these glucosides during the VOO extraction process. Thus, data on olive fruit phenolic composition would be of interest in olive breeding programs with the aim of obtaining new cultivars with improved functional quality. Taking into account that the main phenolics in olive fruits are essentially similar to those found in olive leaves, the objective of the present work was to carry out a comparative study of the phenolic fraction of fruits and leaves in selected cultivars characterized by having different contents of phenolic compounds in the oils to find out if the early selection of olive seedlings could be possible through an analysis of the phenolic compounds of their leaves. Results of the study showed that there is no correlation between the level of the fruit and leaf phenolics for the different olive cultivars. In addition, no significant correlation was found between the content of tyrosol and hydroxytyrosol derivatives in the leaves of the different olive cultivars over time as it does in the case of the fruits during the ripening process. These preliminary data pointed out that although the biosynthesis of the major olive phenolics is carried out through similar biochemical pathways, they seem to be regulated differently in the different organs of the plant.
\end{abstract}

Keywords: Olea europaea L., leaf, fruit, phenolics, breeding

\section{INTRODUCTION}

Virgin olive oil (VOO) represents one of the main features that distinguish the Mediterranean diet. Although different compounds have been shown to have significant health properties such as squalene, triterpenic acids or tocopherols, in particular, the phenolic fraction of VOO has received recent attention because of its benefits to human health (Estruch et al., 2013; Konstantinidou et al., 2010). In this sense, the long term dietary consumption of VOO rich in phenolics seems to be related to an attenuation of the inflammatory response and reduction of the associated risk of chronic inflammatory disease states (Lucas et al., 2011; Visioli and Bernardini, 2011). Besides, phenolics are also

\footnotetext{
${ }^{a}$ E-mail: carlos.sanz@ig.csic.es
} 
responsible for the bitter and pungent taste notes of VOO (Andrewes et al., 2003; Mateos et al., 2004).

The presence of phenolic compounds in VOO is based mainly on the content of phenolic glucosides initially present in the olive fruit tissues and on the activity of hydrolytic and oxidative enzymes acting on these glucosides during the VOO extraction process (García-Rodríguez et al., 2011; Romero-Segura et al., 2009; Romero-Segura et al., 2012). The most abundant VOO phenolics were the secoiridoid derivatives resulting from the hydrolysis of oleuropein, ligstroside and demethyloleuropein present in the fruit, which were on average their main phenolic glucosides. The fruit phenolic content seems to be the main factor determining the synthesis of VOO phenolics during oil extraction according to our recent investigations (García-Rodríguez et al., 2017).

Olive breeding has traditionally focused on the agronomic traits but the increasing number of scientific proofs supporting the positive impact of VOO consumption on human health has made the functional quality of this product to be considered as an olive breeding target. Therefore, phenolic compounds are currently used as quality markers for VOO and also as a trait in new breeding programs (León et al., 2011; El Riachy et al., 2012). Taking into account that the main phenolics in olive fruits are essentially similar to those found in olive leaves (Benavente-García, 2000), the objective of the present work was to carry out a comparative study of the phenolic fraction of fruits and leaves in selected cultivars characterized by having different contents of phenolic compounds in the fruits and oils to find out if the early selection of olive seedlings could be possible through an analysis of the phenolic compounds of their leaves.

\section{MATERIALS AND METHODS}

\section{Plant material}

Olive cultivars characterized by having high (Dokkar, Piñonera), medium (Picual, Arbequina, Menya) and low (Shengeh, Abou kanani) levels of phenolics in the fruit and the oils were studied. Trees, two per accession, were grown in the same edaphoclimatic conditions at the experimental orchards of Instituto de la Grasa (Seville, Spain) using drip irrigation and standard culture practices. Olive leaves were collected in the blooming and fruit lignification period (April-July) from the first, fifth and the leaf pair after first branch (Figure 1) to compare the phenolics profiles. Besides, leaves from the fifth pair and fruits were gathered along the fruit ripening period (July-November) to study their relationship in terms of the phenolics composition. Five different development/ripening stages were chosen: 0, fruits with just lignified stone; I, green fruits; II, green-yellow fruits; III, turning stage, fruits $c a$. $50 \%$ colored; IV, total colored fruits.

\section{Extraction and analysis of olive leaf and fruit phenolic compounds}

Phenolic compounds were extracted from olive leaves and fruits according to RomeroSegura et al. (2012) with some modifications. Transversal pieces (1 mm wide) from leaves and longitudinal pieces of mesocarp tissue from 20 olive leaves and fruits, respectively, were submerged for $72 \mathrm{~h}$ at $4{ }^{\circ} \mathrm{C}$ in dimethyl sulphoxide $(6 \mathrm{ml} / \mathrm{g}$ tissue), containing syringic acid as internal standard $(24 \mathrm{mg} / \mathrm{ml})$. Phenolics extracts were then centrifuged at $3000 \mathrm{~g}$ for $2 \mathrm{~min}$ and filtered through $0.45 \mu \mathrm{m}$ before HPLC analysis.

Phenolic extracts were analyzed in a Beckman Coulter liquid chromatographic system equipped with a diode array detector (System Gold 168) and a Mediterranean Sea 18 column ( $4.0 \mathrm{~mm}$ i.d. $250 \mathrm{~mm}$, particle size $5 \mu \mathrm{m}$ ) (Teknokroma, Barcelona, Spain). Phenolics were monitored at two different wavelengths $280 \mathrm{~nm}$ and $335 \mathrm{~nm}$ and quantified taking into account the internal standard and calibration curves for each of them. The identity of phenolic compounds was established based on their UV-Vis spectra and confirmed by HPLC/ESI-qTOF-HRMS using available standards. For the latter, a Dionex Ultimate 3000 RS U-HPLC liquid chromatograph system (Thermo Fisher Scientific, Waltham, MA, USA) was used, equipped with a similar Mediterranean Sea 18 column but with $3 \mu \mathrm{m}$ particle size. Formic acid $1 \%$ was used instead of phosphoric acid $0.5 \%$ for the mobile phase. A split post- 
column of $0.4 \mathrm{~mL} / \mathrm{min}$ was introduced directly on the mass spectrometer electrospray ion source. The HPLC/ESI-qTOF operated for mass analysis using a micrOTOF-QII High Resolution Time-of-Flight mass spectrometer (UHRTOF) with qQ-TOF geometry (Bruker Daltonics, Bremen, Germany) equipped with an electrospray ionization (ESI) interface. Mass spectra were acquired in MS fullscan mode and data were processed using TargetAnalysis 1.2 software (Bruker Daltonics, Bremen, Germany).

Phenolics were clustered into different groups as follows: Hydroxytyrosol (HTyr) derivatives: hydroxytyrosol-4-glucoside, oleuropein and verbascoside; Tyrosol (Tyr) derivatives: tyrosol-4-glucoside and ligstroside; and Flavonoids: rutin, luteolin-7-glucoside and apigenin-7-glucoside.

\section{RESULTS AND DISCUSSION}

Phenolics profiles of different pairs of leaves were studied in order to select the more suitable for the comparison with phenolic contents of olive fruits. Figure 1 shows the total phenolics contents during the blooming and stone lignification period (April-July) in seven cultivars characterized for featuring different phenolics contents in the fruit and the derived VOO. It was found that most of the leaf phenolics are hydroxytyrosol derivatives in good agreement with Benavente-García et al. (2000). They comprised on average $90 \%$ of total phenolics in the leaves of the seven olive cultivars under study, being oleuropein by far the most abundant phenolic. As shown in Figure 1, the leaves in the different positions of the branch contained on average around $71 \mathrm{mg} / \mathrm{g}$ leaf of phenolics compounds. In general, an increase in the level of phenolics was measured in June, which averaged around $40 \%$, in good agreement to what observed by Blasi et al. (2016). Apparently, the phenol storage in the leaves is a time-dependent regulated process, according to the life cycle of olive leaves as proposed by different authors (Brahmi et al., 2012; Brahmi et al., 2015). However, a different content evolution was monitored along time according to the leaf position. Thus, the June increase is more notable in the leaves from the first pair and then in the $5^{\text {th }}$ pair, being more stable in the pair after the first branch. Another important difference of the phenolic profile of leaves from the first pair is the relatively higher content of tyrosol derivatives compared to the other pair leaves considered. Conversely, the phenolic profile of the leaves from $5^{\text {th }}$ pair and the pair after the first branch are more similar to those found in the fruits. According to these results, the leaves from the $5^{\text {th }}$ pair were chosen to be compared to the phenolic contents of the fruits along the different ripening stages.

Figure 2 displays the content of HTyr derivatives along the fruit development and ripening period both in the $5^{\text {th }}$ pair leaves and fruits. As seen, the leaves of the of olive cultivars characterized by having high levels of phenolics both in the fruit and in their corresponding oils (Dokkar, Piñonera) suffer an increase in the content of these phenolics during the summer, contrary to the rest of the cultivars studied with medium and low phenolics contents in the fruit and oils. As a result, the content of HTyr derivatives was very similar in the leaves and fruits when the fruit begins the ripening process (stage I) and maintain a quite constant level along the ripening period. In the case of the rest of the cultivars studied, in general there was a decrease in the content of derivatives in the fruit during ripening, more pronounced than in the previous ones, while the phenolic contents in the leaves remained fairly constant. They even increased in the last stage of maturation (IV). The case of cultivars characterized by having low levels of phenolic compounds in fruits and oils (Shengeh, Abou kanani) was particularly striking since they have levels of HTyr derivatives in the leaves comparable to those observed in those cultivars that have high levels of these phenolics (Dokkar, Piñonera). No correlation was found between the HTyr derivatives in the fruit and the leaves, either individually or as a sum of them.

On the other hand, the evolution of the content of Tyr derivatives is presented in Figure 3. As shown, the content of these phenolics in the leaves was quite stable throughout the period of development/ripening of the fruit, presenting fairly similar levels around 4500 $\mu \mathrm{g} / \mathrm{g}$ leaf. Apparently, the differences in the contents are not related to the greater or lesser content of Tyr derivatives in the fruits. Thus, the highest average values were found for the cultivar Shengeh (6929 $\mathrm{\mu g} / \mathrm{g}$ leaf), which is characterized by having low levels of these 
derivatives in the fruits. Besides, the levels of Tyr derivatives in the fruits, contrary to the HTyr derivatives, were clearly higher to that presented by the leaves in those cultivars characterized as being rich in phenolics and fruits and oils. As a consequence of these results, no correlation was found between the Tyr derivatives in the fruit and the leaves.

A notable difference was found in the metabolism of HTyr derivatives in the different plant organs. Thus, while there was a good correlation between the level of HTyr and Tyr derivatives in the fruits $(r=0.841, p<0.001)$, there was no correlation between the content of these compounds in the leaves $(r=-0.069)$. Data suggest that there is a parallel synthesis of HTyr and Tyr derivatives in the fruits, as would be expected given the similarity of the molecules, but a complete decoupling in the synthesis of HTyr and Tyr derivatives in the leaves, indicating a separate regulation of the genes that intervene in their synthesis.

Finally, the content of flavonoids remained approximately constant throughout the fruit development/ripening in the different organs (Figure 4). In general, a lower level of flavonoids was found in fruits than in leaves, except in the case of cultivar Piñonera, which was the reverse. A positive significant correlation, although moderate, was found between the content of flavonoids in the leaves and in the fruits $(r=0.389, p<0.05)$. Unexpectedly, the content of flavonoids in the leaves were also correlated, even at a higher level, with the contents of the HTyr derivatives $(r=0.648, p<0.001)$ and Tyr derivatives $(r=0.507, p<0.01)$ in the fruits, but curiously no with these phenolics in the leaves.

\section{CONCLUSIONS}

Results showed that there is no correlation between the level of the fruit and leaf phenolics for the different olive cultivars under study. In addition, no significant correlation was found between the content of tyrosol and hydroxytyrosol derivatives in the leaves of the different olive cultivars over time as it does in the case of the fruits during the ripening process. These preliminary data point out that although the biosynthesis of the major olive phenolics is carried out through similar biochemical pathways, they seem to be regulated differently in the different organs of the plant.

\section{ACKNOWLEDGEMENTS}

Funding for this research came from the project AGL2015-67652 by Programa Nacional de Recursos y Tecnologías Agroalimentarias financed by the Spanish Government.

\section{Literature cited}

Andrewes, P., Busch, J.L.H.C., de Joode, T., Groenewegen, A., and Alexandre, H. (2003). Sensory properties of virgin olive oil polyphenols: identification of deacetoxy-ligstroside glycon as a key contributor to pungency. J. Agric. Food Chem., 51, 1415-1420. doi: 10.1021/jf026042j

Benavente-García, O., Castillo, J., Lorente, J., Ortuño, A., and Del Rio, J.A. (2000). Antioxidant activity of phenolics extracted from Olea europaea L. leaves. Food Chem., 68, 457-462. doi: 10.1016/S0308-8146(99)00221-6

Blasi, F., Urbani, E., Simonetti, M.S., Chiesi, C., Cossignani, L. (2016). Seasonal variations in antioxidant compounds of Olea europaea leaves collected from different Italian cultivars. J. Appl. Bot. Food Qual., 89, 202207. doi: 10.5073/JABFQ.2016.089.025

Brahmi, F., Mechri, B., Dabbou, S., Dhibi, M., and Hammami M. (2012). The efficacy of phenolic compounds with different polarities as antioxidants from olive leaves depending on seasonal variations. Ind. Crop. Prod., 38, 146152. doi: $10.1016 /$ j.indcrop.2012.01.023

Brahmi, F., Mechri, B., Dhibi, M., Hammami M. (2015). Effect of growth stage and solvent extract on the antioxidant potential of olive leaves. J. Plant Sci.,3, 1-7. doi: 10.11648/j.jps.s.2015030301.11

El Riachy, M., Priego-Capote, F., Rallo, L., Luque de Castro, M.D., and León, L. (2012). Phenolic composition of virgin olive oils from cross breeding segregating populations. Eur. J. Lipid Sci. Technol., 114, 542-551. doi: 10.1002/ejlt.201100129

Estruch, R., Ros, E., Salas-Salvadó, J., Covas, M.I., Corella, D., et al. (2013). Primary prevention of cardiovascular disease with a Mediterranean diet. N. Engl. J. Med., 368, 1279-1290. doi: 10.1056/NEJMoa1200303 
García-Rodríguez, R., Belaj, A., Romero-Segura, C., Sanz, C., and Pérez, A. (2017). Exploration of genetic resources to improve the functional quality of virgin olive oil. J. Funct. Foods, 38, 1-8. doi: 10.1016/j.jff.2017.08.043

García-Rodríguez, R., Romero-Segura, C., Sanz, C., Sánchez-Ortiz, A., and Pérez, A. (2011). Role of polyphenol oxidase and peroxidase in shaping the phenolic profile of virgin olive oil. Food Res. Int., 44, 265-635. doi: 10.1016/j.foodres.2010.12.023

Konstantinidou, V., Covas, M.I., Muñoz-Aguayo, D., Khymenets, O., de La Torre, R., et al. (2010). In vivo nutrigenomic effects of VOO polyphenols within the frame of the Mediterranean diet: a randomized trial. Faseb. J., 24, 2546-2557. doi: 10.1096/fj.09-148452

León, L., Beltrán, G., Aguilera, M. P., Rallo, L., Barranco, D., and de la Rosa, R. (2011). Oil composition of advanced selections from an olive breeding program. Eur. J. Lipid Sci. Technol., 113, 870-875. doi: 10.1002/ejlt.201000535

Lucas, L., Russell, A., and Keast, R. (2011). Molecular mechanisms of inflammation. Anti-inflammatory benefits of virgin olive oil and the phenolic compound oleocanthal. Curr. Pharm. Design., 17, 754-768. doi: $10.2174 / 138161211795428911$

Mateos, R., Cert, A., Pérez-Camino, M.C., and García, J.M. (2004). Evaluation of virgin olive oil bitterness by quantification of secoiridoid derivatives. J. Am. Oil. Chem. Soc., 81, 71-75. doi: 10.1007/s11746-004-0859-x

Romero-Segura, C., García-Rodríguez, R., Sánchez-Ortiz, A., Sanz, C., and Pérez, A.G. (2012). The role of olive $\beta$ glucosidase in shaping the phenolic profile of virgin olive oil. Food Res. Int., 45, 191-196. doi: 10.1016/j.foodres.2011.10.024

Romero-Segura, C., Sanz, C., and Pérez, A.G. (2009). Purification and characterization of an olive fruit $\beta$ glucosidase involved in the biosynthesis of virgin olive oil phenolics. J. Agric. Food Chem., 57, 7983-7988. doi: 10.1021/jf901293c

Visioli, F., and Bernardini, E. (2011). Extra virgin olive oil's polyphenols: Biological activities. Curr. Pharm. Design., 17, 786-804. doi: 10.2174/138161211795428885
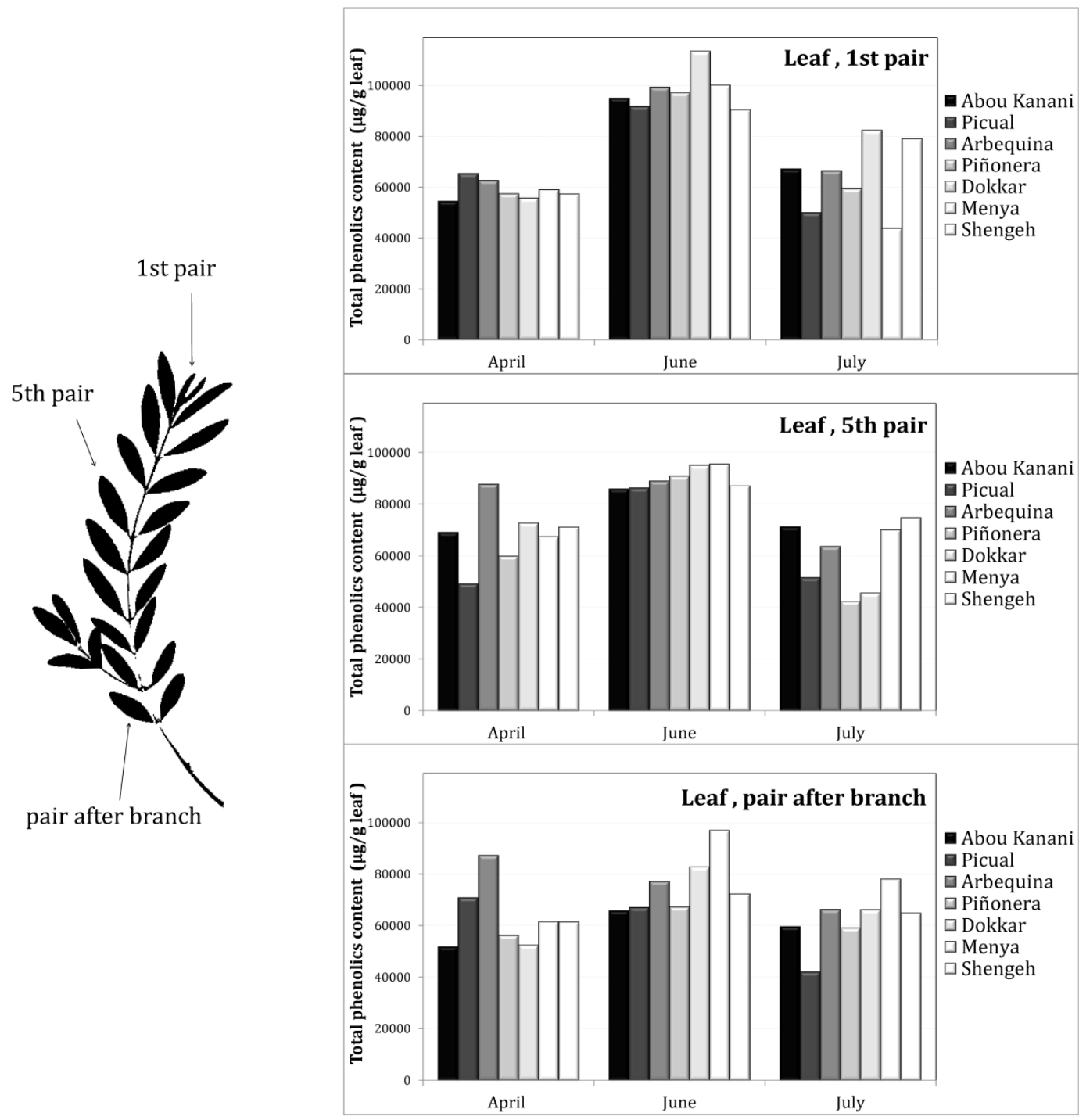
Figure 1. Evolution of total content of phenolic compounds ( $\mu \mathrm{g} / \mathrm{g}$ leaf) during the blooming and stone lignification period in different pairs of leaves of selected cultivars.

Hydroxytyrosol derivatives
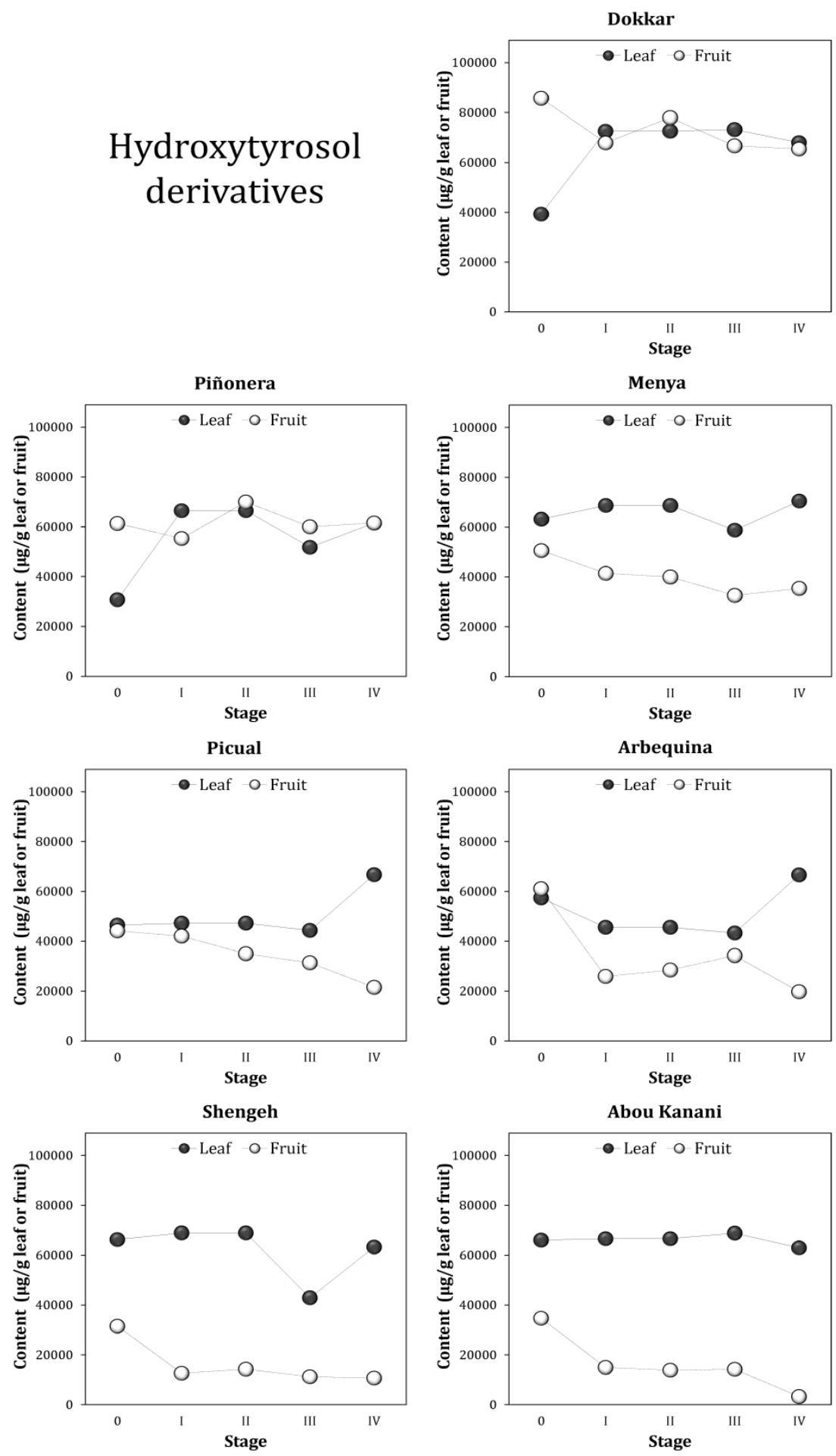
Figure 2. Evolution of the content of hidroxytyrosol derivatives ( $\mu \mathrm{g} / \mathrm{g}$ leaf or fruit) in leaf and fruit during the development/ripening of fruit of the seven cultivars under study.
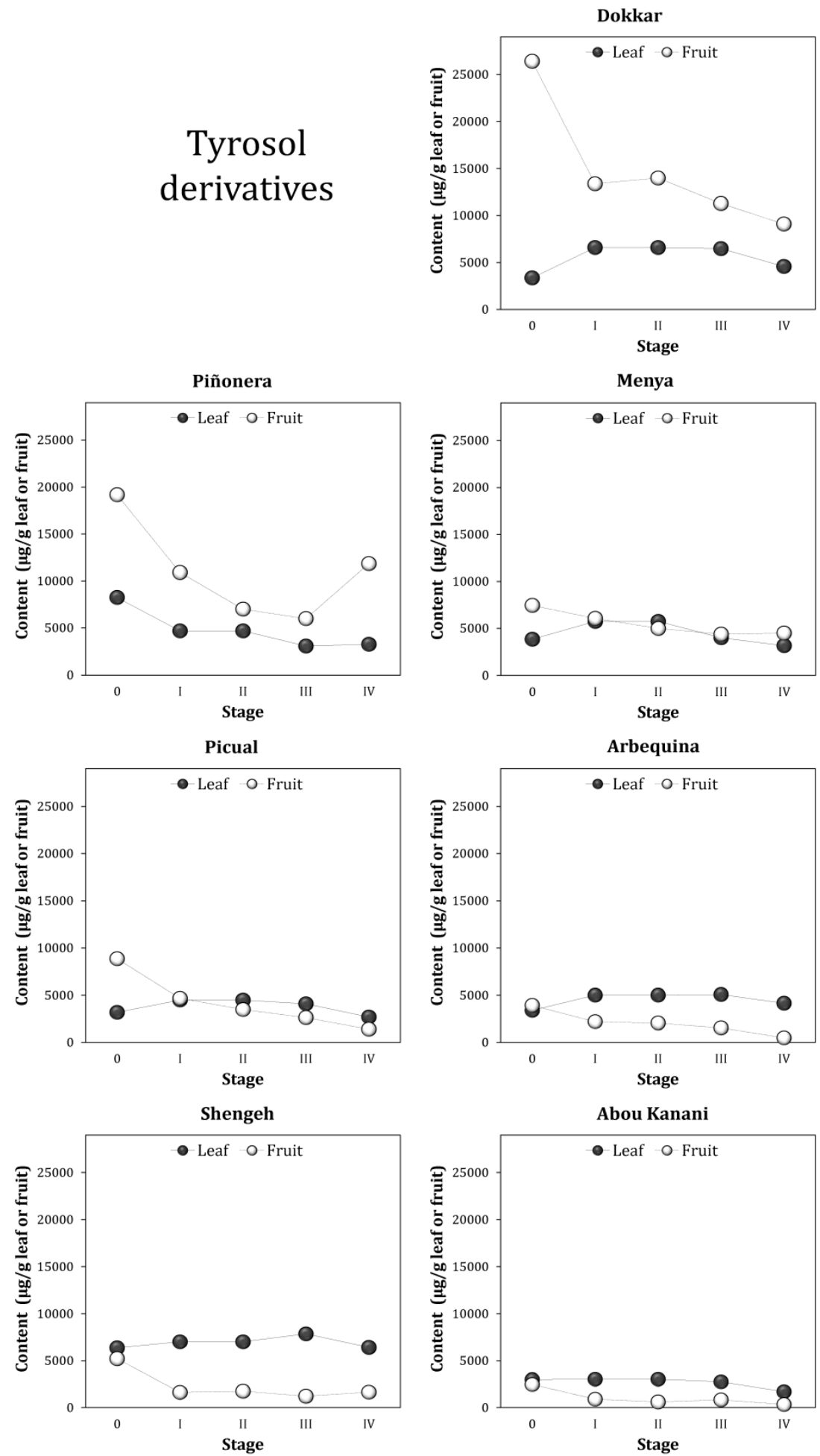
Figure 3. Evolution of the content of tyrosol derivatives ( $\mu \mathrm{g} / \mathrm{g}$ leaf or fruit) in leaf and fruit during the development/ripening of fruit of the seven cultivars under study.
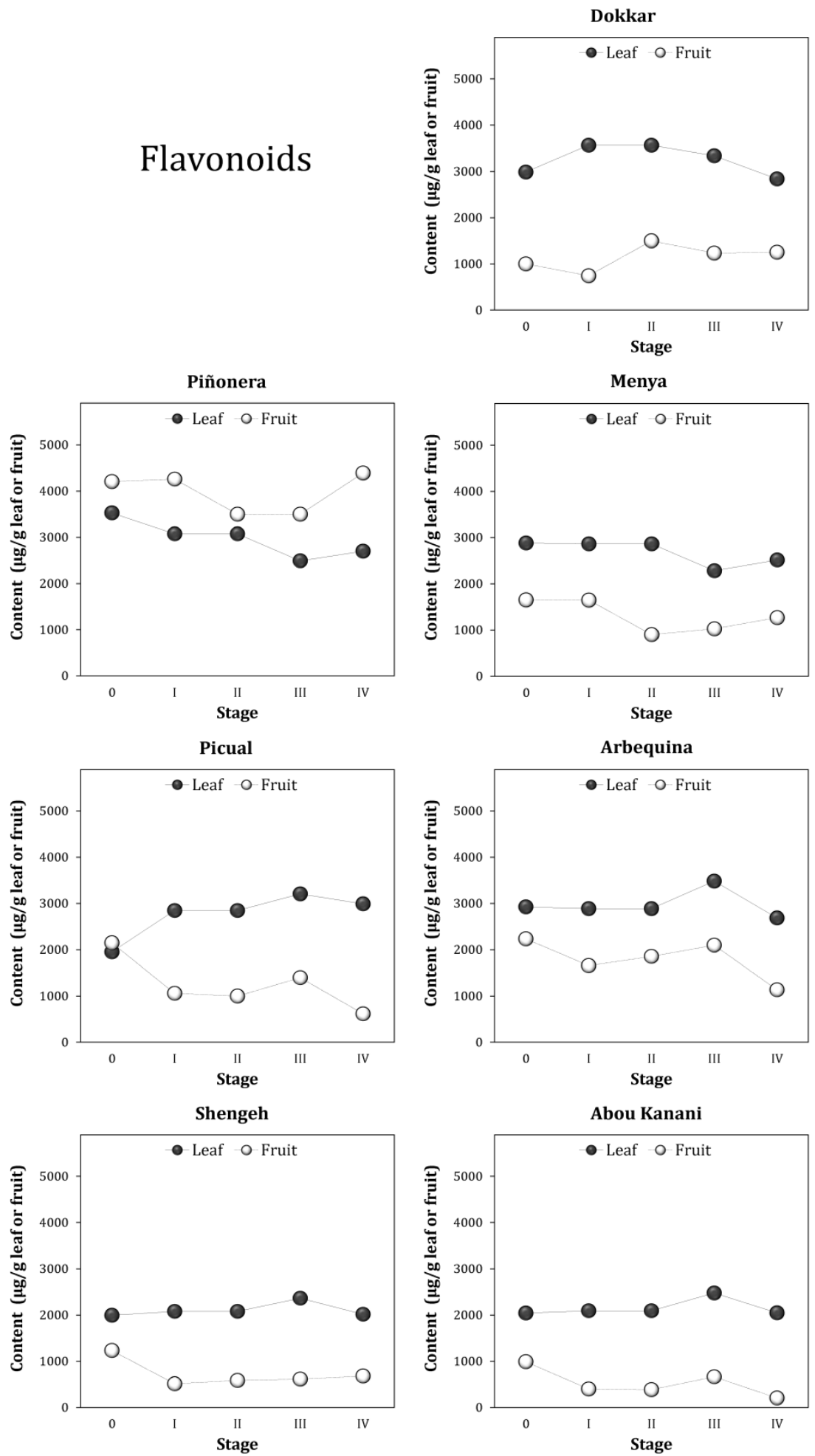
Figure 4. Evolution of the content of flavonoids ( $\mu \mathrm{g} / \mathrm{g}$ leaf or fruit) in leaf and fruit during the development/ripening of fruit of the seven cultivars under study. 